\title{
Existence and uniqueness of positive solutions for a new class of coupled system via fractional derivatives
}

\author{
Hojjat Afshari ${ }^{1 *}$, Mojtaba Sajjadmanesh ${ }^{1}$ and Dumitru Baleanu ${ }^{2,3}$
}

"Correspondence:
hojat.afshari@yahoo.com
${ }^{1}$ Department of Mathematics,
Faculty of Basic Science, University
of Bonab, Bonab, Iran
Full list of author information is
available at the end of the article

\section{Springer}

\begin{abstract}
In this paper we study the existence of unique positive solutions for the following coupled system:

$$
\left\{\begin{array}{l}
D_{0^{+}}^{\alpha} x(\tau)+f_{1}\left(\tau, x(\tau), D_{0^{+}}^{\eta} x(\tau)\right)+g_{1}(\tau, y(\tau))=0 \\
D_{0^{+}}^{\beta} y(\tau)+f_{2}\left(\tau, y(\tau), D_{0^{+}}^{\gamma} y(\tau)\right)+g_{2}(\tau, x(\tau))=0 \\
\tau \in(0,1), \quad n-1<\alpha, \beta<n \\
x^{(i)}(0)=y^{(i)}(0)=0, \quad i=0,1,2, \ldots, n-2 ; \\
{\left[D_{0^{+}}^{\xi} y(\tau)\right]_{\tau=1}=k_{1}(y(1)), \quad\left[D_{0^{+}}^{\zeta} x(\tau)\right]_{\tau=1}=k_{2}(x(1))}
\end{array}\right.
$$

where the integer number $n>3$ and $1 \leq \gamma \leq \xi \leq n-2,1 \leq \eta \leq \zeta \leq n-2$, $f_{1}, f_{2}:[0,1] \times \mathbb{R}^{+} \times \mathbb{R}^{+} \rightarrow \mathbb{R}^{+}, g_{1}, g_{2}:[0,1] \times \mathbb{R}^{+} \rightarrow \mathbb{R}^{+}$and $k_{1}, k_{2}: \mathbb{R}^{+} \rightarrow \mathbb{R}^{+}$are continuous functions, $D_{0^{+}}^{\alpha}$ and $D_{0^{+}}^{\beta}$ stand for the Riemann-Liouville derivatives. An illustrative example is given to show the effectiveness of theoretical results.
\end{abstract}

Keywords: Fractional differential equation; Mixed monotone operator; Normal cone; Coupled system

\section{Introduction}

A lot of fractional differential equations and coupled systems have been studied widely; see $[1-19,24]$ and the references therein. As is well known, coupled systems with boundary conditions appear in the investigations of many problems such as mathematical biology (see $[9,30])$, natural sciences and engineering; for example, we can see beam deformation and steady-state heat flow (see $[25,26]$ ) and heat equations (see $[18,24]$ ). So the subject of coupled systems is gaining much attention and importance. There are a large number of articles dealing with the existence or multiplicity of solutions or positive solutions for some nonlinear coupled systems with boundary conditions; for details, see [7, 8, 10, 11, $20,21,27,29,32,33,35-41]$.

(0) The Author(s) 2020. This article is licensed under a Creative Commons Attribution 4.0 International License, which permits use, sharing, adaptation, distribution and reproduction in any medium or format, as long as you give appropriate credit to the original author(s) and the source, provide a link to the Creative Commons licence, and indicate if changes were made. The images or other third party material in this article are included in the article's Creative Commons licence, unless indicated otherwise in a credit line to the material. If material is not included in the article's Creative Commons licence and your intended use is not permitted by statutory regulation or exceeds the permitted use, you will need to obtain permission directly from the copyright holder. To view a copy of this licence, visit http://creativecommons.org/licenses/by/4.0/. 
In [42] Zhang and Tian considered a unique positive solution for the following problem:

$$
\left\{\begin{array}{l}
D_{0^{+}}^{\alpha} w(\tau)+f\left(\tau, w(\tau), D_{0^{+}}^{\gamma} w(\tau)\right)+g(\tau, w(\tau))=0, \quad \tau \in(0,1), n-1<\alpha<n \\
w^{(i)}(0)=0, \quad i=0,1,2, \ldots, n-2 \\
{\left[D_{0^{+}}^{\beta} w(\tau)\right]_{\tau=1}=k(w(1))}
\end{array}\right.
$$

where $n>3,1 \leq \gamma \leq \beta \leq n-2, f:[0,1] \times \mathbb{R}^{+} \times \mathbb{R}^{+} \rightarrow \mathbb{R}^{+}, g:[0,1] \times \mathbb{R}^{+} \rightarrow \mathbb{R}^{+}$and $k:$ $\mathbb{R}^{+} \rightarrow \mathbb{R}^{+}$are continuous functions, $D_{0^{+}}^{\alpha}$ is the Riemann-Liouville fractional derivative and $w^{(i)}$ represents the $i$ th (ordinary) derivative of $w$.

Continuing their work, we establish the existence of solutions for the following coupled system:

$$
\left\{\begin{array}{l}
D_{0^{+}}^{\alpha} x(\tau)+f_{1}\left(\tau, x(\tau), D_{0^{+}}^{\eta} x(\tau)\right)+g_{1}(\tau, y(\tau))=0, \\
D_{0^{+}}^{\beta} y(\tau)+f_{2}\left(\tau, y(\tau), D_{0^{+}}^{\gamma} y(\tau)\right)+g_{2}(\tau, x(\tau))=0, \quad \tau \in(0,1), n-1<\alpha, \beta<n ; \\
x^{(i)}(0)=y^{(i)}(0)=0, \quad i=0,1,2, \ldots, n-2 ; \\
{\left[D_{0^{+}}^{\xi} y(\tau)\right]_{\tau=1}=k_{1}(y(1)), \quad\left[D_{0^{+}}^{\zeta} x(\tau)\right]_{\tau=1}=k_{2}(x(1)),}
\end{array}\right.
$$

where the integer number $n>3$ and $1 \leq \gamma \leq \xi \leq n-2,1 \leq \eta \leq \zeta \leq n-2, f_{1}, f_{2}:[0,1] \times$ $\mathbb{R}^{+} \times \mathbb{R}^{+} \rightarrow \mathbb{R}^{+}, g_{1}, g_{2}:[0,1] \times \mathbb{R}^{+} \rightarrow \mathbb{R}^{+}$and $k_{1}, k_{2}: \mathbb{R}^{+} \rightarrow \mathbb{R}^{+}$are continuous functions, $D_{0^{+}}^{\alpha}$ and $D_{0^{+}}^{\beta}$ stand for the Riemann-Liouville derivatives.

\section{Preliminaries}

Suppose $(E,\|\cdot\|)$ is a Banach space which is partially ordered by a cone $P \subseteq E$. We denote the zero element of $E$ by $\theta$. A cone $P$ is called normal if there exists a constant $N>0$ such that $\theta \leq x \leq y$ implies $\|x\| \leq N\|y\|$.

Definition 2.1 ([22, 23]) $A: P \times P \rightarrow P$ is said to be a mixed monotone operator if $A(x, y)$ is increasing in $x$ and decreasing in $y$, i.e., for $x_{i}, y_{i} \in P(i=1,2), x_{1} \leq x_{2}, y_{1} \geq y_{2}$ imply $A\left(x_{1}, y_{1}\right) \leq A\left(x_{2}, y_{2}\right)$. The element $x \in P$ is called a fixed point of $A$ if $A(x, x)=x$.

An element $u^{*} \in D$ is called a fixed point of $A$ if it satisfies $A\left(u^{*}, u^{*}\right)=u^{*}$. Let $h>\theta$, write $P_{h}=\{u \in E \mid \exists \lambda, \mu>0: \lambda h \leq u \leq \mu h\}$.

Let $\Phi$ be a class of functions $\varphi:(0,1) \rightarrow(0,1)$ with $\varphi(\tau)>\tau$ for $\tau \in(0,1)$.

Theorem 2.2 ([34]) Let $P$ be a normal cone in $E, \alpha \in(0,1) A: P \rightarrow P$ is an increasing sub-homogeneous, $B: P \rightarrow P$ is a decreasing operator, $C: P \times P \rightarrow P$ is a mixed monotone operator and that satisfy the following conditions:

$$
B\left(\frac{1}{\tau} u\right) \geq \tau B u, \quad C\left(\tau u, \frac{1}{\tau} v\right) \geq \tau^{\alpha} C(u, v), \quad u, v \in P .
$$

\section{Assume that}

(i) $\exists h_{0} \in P_{h}$ such that $A h_{0} \in P_{h}, B h_{0} \in P_{h}, C\left(h_{0}, h_{0}\right) \in P_{h}$;

(ii) $\exists \delta_{0}>0$ with $C(u, v) \geq \delta_{0}(A u+B v)$ for $u, v \in P$.

Then

(1) $A: P_{h} \rightarrow P_{h}, B: P_{h} \rightarrow P_{h}$ and $C: P_{h} \times P_{h} \rightarrow P_{h}$; 
(2) $\exists x_{0}, y_{0} \in P_{h}$ and $r \in(0,1)$ with

$$
r x_{0} \leq x_{0}<y_{0}, x_{0} \leq A x_{0}+B y_{0}+C\left(x_{0}, y_{0}\right) \leq A y_{0}+B x_{0}+C\left(y_{0}, x_{0}\right) \leq y_{0}
$$

(3) the equation $A u+B u+C(u, u)=u$ has a unique solution $u^{*}$ in $P_{h}$;

(4) for $x_{0}, y_{0} \in P_{h}$, we can construct

$$
\begin{aligned}
& u_{n}=A x_{n-1}+B y_{n-1}+C\left(x_{n-1}, y_{n-1}\right) \text {, } \\
& v_{n}=A y_{n-1}+B x_{n-1}+C\left(y_{n-1}, x_{n-1}\right), \quad n=1,2, \ldots \\
& \text { and } u_{n} \rightarrow u^{*} \text { and } v_{n} \rightarrow v^{*} \text {. }
\end{aligned}
$$

Definition 2.3 ([28, 31]) The Riemann-Liouville fractional derivative for a continuous function $f$ is defined by

$$
D^{\alpha} f(\tau)=\frac{1}{\Gamma(n-\alpha)}\left(\frac{d}{d \tau}\right)^{n} \int_{0}^{\tau} \frac{f(\rho)}{(t-\rho)^{\alpha-n+1}} d \rho \quad(n=[\alpha]+1)
$$

where the right-hand side is point-wise defined on $(0, \infty)$.

Definition 2.4 ([28, 31]) Let $[a, b]$ be an interval in $\mathbb{R}$ and $\alpha>0$. The Riemann-Liouville fractional order integral of a function $f \in L^{1}([a, b], \mathbb{R})$ is defined by

$$
I_{a}^{\alpha} f(\tau)=\frac{1}{\Gamma(\alpha)} \int_{a}^{\tau} \frac{f(\rho)}{(\tau-\rho)^{1-\alpha}} d \rho,
$$

whenever the integral exists.

Lemma $2.5([42])$ Let $h \in C[0,1]$, then the unique solution of the linear problem

$$
\begin{aligned}
& D_{0^{+}}^{\alpha} x(\tau)+h(\tau)=0, \quad \tau \in(0,1), n-1<\alpha \leq n ; \\
& x^{i}(0)=0, \quad i=0,1,2,3, \ldots, n-2 ; \\
& {\left[D_{0^{+}}^{\beta} x(\tau)\right]_{\tau=1}=k(x(1)), \quad 1 \leq \beta \leq n-2 ;}
\end{aligned}
$$

is given by

$$
x(\tau)=\int_{0}^{1} G(\tau, \rho) h(\rho) d \rho+\frac{\Gamma(\alpha-\beta)}{\Gamma(\alpha)} k(x(1)) \tau^{\alpha-1},
$$

where

$$
G(\tau, \rho)= \begin{cases}\frac{\tau^{\alpha-1}(1-\rho)^{\alpha-\beta-1}-(\tau-\rho)^{\alpha-1}}{\Gamma(\alpha)}, & 0 \leq \rho \leq \tau \leq 1 \\ \frac{\tau^{\alpha-1}(1-\rho)^{\alpha-\beta-1}}{\Gamma(\alpha)}, & 0 \leq \tau \leq \rho \leq 1\end{cases}
$$

is the Green function. 
Lemma 2.6 ([42]) The Green function (7) has the following properties:

$$
\begin{aligned}
0 & \leq \tau^{\alpha-1}(1-\rho)^{\alpha-\beta-1}\left[1-(1-\rho)^{\beta}\right] \leq \Gamma(\alpha) G(\tau, \rho) \leq \tau^{\alpha-1}(1-\rho)^{\alpha-\beta-1}, \\
0 & \leq \tau^{\alpha-\gamma-1}(1-\rho)^{\alpha-\beta-1}\left[1-(1-\rho)^{\beta-\gamma}\right] \leq \Gamma(\alpha-\gamma) D_{0^{+}}^{\gamma} G(\tau, \rho) \\
& \leq \tau^{\alpha-\gamma-1}(1-\rho)^{\alpha-\beta-1}, \quad \tau, \rho \in[0,1] .
\end{aligned}
$$

Lemma 2.7 ([36]) $K_{h}=P_{h_{1}} \times P_{h_{2}}$, where that $K=P \times P$ and $h(\tau)=\left(h_{1}, h_{2}\right)$.

\section{Main results}

Let $E \times E \subset X \times X$ with $X=C[0,1]$ such that $E=\left\{x \mid x, D_{0^{+}}^{\eta} x, D_{0^{+}}^{\gamma} x \in X\right\}$ endowed with the norm $\|x\|=\max \left\{\max _{\tau \in[0,1]}|x(\tau)|, \max _{\tau \in[0,1]} D_{0^{+}}^{\eta}|x(\tau)|, \max _{\tau \in[0,1]} D_{0^{+}}^{\gamma}|x(\tau)|\right\}$. For $(x, y) \in$ $E \times E$, let $\|(x, y)\|=\max \{\|x\|,\|y\|\}$. It is easy to see that $(E \times E,\|(x, y)\|)$ is a Banach space. Define $P=\left\{x \in E: x, D_{0^{+}}^{\eta} x, D_{0^{+}}^{\gamma} x \geq 0\right\}, K=P \times P$, then $K$ is a normal cone equipped with the following partial order:

$$
\left(x_{1}, y_{1}\right) \preceq\left(x_{2}, y_{2}\right) \quad \Leftrightarrow \quad x_{1} \leq x_{2}, y_{1} \leq y_{2},
$$

and

$$
\begin{array}{ll}
D_{0^{+}}^{\eta} x_{1}(\tau) \leq D_{0^{+}}^{\eta} x_{2}(\tau), & D_{0^{+}}^{\gamma} x_{1}(\tau) \leq D_{0^{+}}^{\gamma} x_{2}(\tau), \\
D_{0^{+}}^{\eta} y_{1}(\tau) \leq D_{0^{+}}^{\eta} y_{2}(\tau), & D_{0^{+}}^{\gamma} y_{1}(\tau) \leq D_{0^{+}}^{\gamma} y_{2}(\tau) .
\end{array}
$$

By Lemma 2.5 in [42], the unique positive solution for the problem (1) is given by

$$
\begin{aligned}
x(\tau)= & \int_{0}^{1} G(\tau, \rho) f\left(\rho, x(\rho), D_{0^{+}}^{\gamma} x(\rho)\right) d \rho \\
& +\int_{0}^{1} G(\tau, \rho) g(\rho, x(\rho)) d \rho+\frac{\Gamma(\alpha-\beta)}{\Gamma(\alpha)} k(x(1)) \tau^{\alpha-1},
\end{aligned}
$$

where

$$
G(\tau, \rho)= \begin{cases}\frac{\tau^{\alpha-1}(1-\rho)^{\alpha-\beta-1}-(\tau-\rho)^{\alpha-1}}{\Gamma(\alpha)}, & 0 \leq \rho \leq \tau \leq 1 ; \\ \frac{\tau^{\alpha-1}(1-\rho)^{\alpha-\beta-1}}{\Gamma(\alpha)}, & 0 \leq \tau \leq \rho \leq 1,\end{cases}
$$

is a Green function.

Assume that $f_{1}(\tau, x, y), f_{2}(\tau, x, y)$ are continuous, then $(x, y) \in X \times X$ is a solution of the system (2) if and only if $(x, y)$ is a solution of the integral equations

$$
\left\{\begin{aligned}
x(\tau)= & \int_{0}^{1} G_{1}(\tau, \rho) f_{1}\left(\rho, x(\rho), D_{0^{+}}^{\eta} x(\rho)\right) d \rho \\
& +\int_{0}^{1} G_{1}(\tau, \rho) g_{1}(\rho, y(\rho)) d \rho+\frac{\Gamma(\alpha-\zeta)}{\Gamma(\alpha)} k_{2}(x(1)) \tau^{\alpha-1}, \\
y(\tau)= & \int_{0}^{1} G_{2}(\tau, \rho) f_{2}\left(\rho, y(\rho), D_{0^{+}}^{\gamma} y(\rho)\right) d \rho \\
& +\int_{0}^{1} G_{2}(\tau, \rho) g_{2}(\rho, x(\rho)) d \rho+\frac{\Gamma(\beta-\xi)}{\Gamma(\beta)} k_{1}(y(1)) \tau^{\beta-1},
\end{aligned}\right.
$$


where

$$
G_{1}(\tau, \rho)= \begin{cases}\frac{\tau^{\alpha-1}(1-\rho)^{\alpha-\zeta-1}-(\tau-\rho)^{\alpha-1}}{\Gamma(\alpha)}, & 0 \leq \rho \leq \tau \leq 1 \\ \frac{\tau^{\alpha-1}(1-\rho)^{\alpha-\zeta-1}}{\Gamma(\alpha)}, & 0 \leq \tau \leq \rho \leq 1\end{cases}
$$

and

$$
G_{2}(\tau, \rho)= \begin{cases}\frac{\tau^{\beta-1}(1-\rho)^{\beta-\xi-1}-(\tau-\rho)^{\beta-1}}{\Gamma(\beta)}, & 0 \leq \rho \leq \tau \leq 1 \\ \frac{\tau^{\beta-1}(1-\rho)^{\beta-\xi-1}}{\Gamma(\beta)}, & 0 \leq \tau \leq \rho \leq 1\end{cases}
$$

are Green functions.

Let us define the operators $A_{1}, B_{1}, C_{1}, A_{2}, B_{2}, C_{2}$ by

$$
\begin{aligned}
& A_{1}(u)(\tau)=\int_{0}^{1} G_{1}(\tau, \rho) g_{1}(\rho, v(\rho)) d \rho, \quad A_{2}(v)(\tau)=\int_{0}^{1} G_{2}(\tau, \rho) g_{2}(\rho, u(\rho)) d \rho, \\
& B_{1}(u)(\tau)=\frac{\Gamma(\alpha-\zeta)}{\Gamma(\alpha)} k_{2}(u(1)) \tau^{\alpha-1}, \quad B_{2}(v)(\tau)=\frac{\Gamma(\beta-\xi)}{\Gamma(\beta)} k_{1}(v(1)) \tau^{\beta-1}, \\
& C_{1}(v, u)(\tau)=\int_{0}^{1} G_{1}(\tau, \rho) f_{1}\left(\rho, v(\rho), D_{0^{+}}^{\eta} u(\rho)\right) d \rho \\
& C_{2}(u, v)(\tau)=\int_{0}^{1} G_{2}(\tau, \rho) f_{2}\left(\rho, u(\rho), D_{0^{+}}^{\gamma} \nu(\rho)\right) d \rho,
\end{aligned}
$$

for $0 \leq \tau \leq 1$.

\section{Theorem 3.1 Assume that}

$\left(H_{1}\right) f_{1}, f_{2}:[0,1] \times \mathbb{R}^{+} \times \mathbb{R}^{+} \rightarrow \mathbb{R}^{+}, g_{1}, g_{2}:[0,1] \times \mathbb{R}^{+} \rightarrow \mathbb{R}^{+}$and $k_{1}, k_{2}: \mathbb{R}^{+} \rightarrow \mathbb{R}^{+}$are continuous, also: $f_{1}(\tau, 1,0) \not \equiv 0, f_{2}(\tau, 1,0) \not \equiv 0$;

$\left(H_{2}\right) f_{1}(\tau, x, y)$ and $f_{2}(\tau, x, y)$ are increasing respect to $x \in \mathbb{R}^{+}$, decreasing respect to $y \in \mathbb{R}^{+}$, $g_{1}, g_{2}$ are increasing respect to $y$ for fixed $0 \leq \tau \leq 1$ and $k_{1}, k_{2}$ are decreasing with $k_{1}(y(1)), k_{2}(x(1)) \neq 0$;

$\left(H_{3}\right) \exists \alpha_{1}, \alpha_{2} \in(0,1)$ such that

$$
f_{1}\left(\tau, \lambda x, \lambda^{-1} y\right) \geq \lambda^{\alpha_{1}} f_{1}(\tau, x, y), \quad f_{2}\left(\tau, \lambda x, \lambda^{-1} y\right) \geq \lambda^{\alpha_{2}} f_{2}(\tau, x, y),
$$

and $g_{1}, g_{2}, k_{1}, k_{2}$ satisfy

$$
g_{i}(\tau, \lambda x) \geq \lambda g_{i}(\tau, x), \quad k_{i}\left(\lambda^{-1} x\right) \geq \lambda k_{i}(x), \quad i=1,2,
$$

for $\lambda \in(0,1), 0 \leq \tau \leq 1, x \in \mathbb{R}^{+}$

$\left(H_{4}\right) g_{i}(\tau, 0) \not \equiv 0$ and there exist positive constants $\delta_{11}, \delta_{12}, \delta_{21}$ and $\delta_{22}$ such that

$$
\begin{aligned}
& f_{i}(\tau, x, y) \geq \delta_{i 1} g_{i}(\tau, x), \\
& f_{i}(\tau, x, y) \geq \delta_{i 2} \geq k_{i}(y), \quad(i=1,2), 0 \leq \tau \leq 1, x, y \in \mathbb{R}^{+} .
\end{aligned}
$$


(1) $\exists\left(u_{01}, u_{02}\right),\left(v_{01}, v_{02}\right) \in K \subset E \times E$ and $r \in(0,1)$ such that

$$
r\left(v_{01}, v_{02}\right) \preceq\left(u_{01}, u_{02}\right) \prec\left(v_{01}, v_{02}\right),
$$

that is,

$$
\begin{aligned}
& r\left(v_{01}, v_{02}\right) \leq\left(u_{01}, u_{02}\right)<\left(v_{01}, v_{02}\right) \text {, } \\
& r\left(D_{0^{+}}^{\gamma} \nu_{01}, D_{0^{+}}^{\gamma} v_{02}\right) \leq\left(D_{0^{+}}^{\eta} u_{01}, D_{0^{+}}^{\eta} u_{02}\right)<\left(D_{0^{+}}^{\gamma} v_{01}, D_{0^{+}}^{\gamma} v_{02}\right), \\
& \left(u_{01}, u_{02}\right) \leq\left(\int_{0}^{1} G_{1}(\tau, \rho) f_{1}\left(\rho, u_{01}(\rho), D_{0^{+}}^{\eta} u_{01}(\rho)\right) d \rho\right. \\
& +\int_{0}^{1} G_{1}(\tau, \rho) g_{1}\left(\rho, v_{01}(\rho)\right) d \rho+\frac{\Gamma(\alpha-\zeta)}{\Gamma(\alpha)} k_{2}\left(u_{01}(1)\right) \tau^{\alpha-1}, \\
& \int_{0}^{1} G_{1}(\tau, \rho) f_{1}\left(\rho, u_{02}(\rho), D_{0^{+}}^{\eta} u_{02}(\rho)\right) d \rho \\
& \left.+\int_{0}^{1} G_{1}(\tau, \rho) g_{1}\left(\rho, v_{02}(\rho)\right) d \rho+\frac{\Gamma(\alpha-\zeta)}{\Gamma(\alpha)} k_{2}\left(u_{02}(1)\right) \tau^{\alpha-1}\right), \\
& D_{0^{+}}^{\eta}\left(\left(u_{01}, u_{02}\right)\right) \leq\left(\int_{0}^{1} D_{0^{+}}^{\eta} G_{1}(\tau, \rho) f_{1}\left(\rho, u_{01}(\rho), D_{0^{+}}^{\eta} u_{01}(\rho)\right) d \rho\right. \\
& +\int_{0}^{1} D_{0^{+}}^{\eta} G_{1}(\tau, \rho) g_{1}\left(\rho, v_{01}(\rho)\right) d \rho+\frac{\Gamma(\alpha-\zeta)}{\Gamma(\alpha)} k_{2}\left(u_{01}(1)\right) \tau^{\alpha-1}, \\
& \int_{0}^{1} D_{0^{+}}^{\eta} G_{1}(\tau, \rho) f_{1}\left(\rho, u_{02}(\rho), D_{0^{+}}^{\eta} u_{02}(\rho)\right) d \rho \\
& \left.+\int_{0}^{1} D_{0^{+}}^{\eta} G_{1}(\tau, \rho) g_{1}\left(\rho, v_{02}(\rho)\right) d \rho+\frac{\Gamma(\alpha-\zeta)}{\Gamma(\alpha)} k_{2}\left(u_{02}(1)\right) \tau^{\alpha-1}\right), \\
& \left(v_{01}, v_{02}\right) \geq\left(\int_{0}^{1} G_{2}(\tau, \rho) f_{2}\left(\rho, v_{01}(\rho), D_{0^{+}}^{\gamma} v_{01}(\rho)\right) d \rho\right. \\
& +\int_{0}^{1} G_{2}(\tau, \rho) g_{2}\left(\rho, u_{01}(\rho)\right) d \rho+\frac{\Gamma(\beta-\xi)}{\Gamma(\beta)} k_{1}\left(v_{01}(1)\right) \tau^{\beta-1}, \\
& \int_{0}^{1} G_{2}(\tau, \rho) f_{2}\left(\rho, v_{02}(\rho), D_{0^{+}}^{\gamma} v_{02}(\rho)\right) d \rho \\
& \left.+\int_{0}^{1} G_{2}(\tau, \rho) g_{2}\left(\rho, u_{02}(\rho)\right) d \rho+\frac{\Gamma(\beta-\xi)}{\Gamma(\beta)} k_{1}\left(v_{02}(1)\right) \tau^{\beta-1}\right), \\
& D_{0^{+}}^{\gamma}\left(v_{01}, v_{02}\right) \geq\left(\int_{0}^{1} D_{0^{+}}^{\gamma} G_{2}(\tau, \rho) f_{2}\left(\rho, v_{01}(\rho), D_{0^{+}}^{\gamma} v_{01}(\rho)\right) d \rho\right. \\
& +\int_{0}^{1} D_{0^{+}}^{\gamma} G_{2}(\tau, \rho) g_{2}\left(\rho, u_{01}(\rho)\right) d \rho+\frac{\Gamma(\beta-\xi)}{\Gamma(\beta)} k_{1}\left(v_{01}(1)\right) \tau^{\beta-1}, \\
& \int_{0}^{1} D_{0^{+}}^{\gamma} G_{2}(\tau, \rho) f_{2}\left(\rho, v_{02}(\rho), D_{0^{+}}^{\gamma} v_{02}(\rho)\right) d \rho \\
& \left.+\int_{0}^{1} D_{0^{+}}^{\gamma} G_{2}(\tau, \rho) g_{2}\left(\rho, u_{02}(\rho)\right) d \rho+\frac{\Gamma(\beta-\xi)}{\Gamma(\beta)} k_{1}\left(v_{02}(1)\right) \tau^{\beta-1}\right),
\end{aligned}
$$

where $G_{1}(\tau, \rho), G_{2}(\tau, \rho)$ are defined by (11) and (12), respectively. 
(2) The problem (2) has a unique positive solution $\left(u^{*}, v^{*}\right)$ in $K_{h}$, with $h(\tau)=\left(h_{1}(\tau)\right.$, $\left.h_{2}(\tau)\right)=\left(\tau^{\alpha-1}, \tau^{\beta-1}\right), 0 \leq \tau \leq 1$.

(3) For $\left(x_{01}, x_{02}\right),\left(y_{01}, y_{02}\right) \in P_{h} \times P_{h}$, there are two iterative sequences $\left\{\left(x_{n 1}, x_{n 2}\right)\right\}$, $\left\{\left(y_{n 1}, y_{n 2}\right)\right\}$ for approximating $\left(x^{*}, y^{*}\right)$, that is, $\left(x_{n 1}, x_{n 2}\right) \rightarrow\left(x^{*}, y^{*}\right),\left(y_{n 1}, y_{n 2}\right) \rightarrow\left(x^{*}, y^{*}\right)$, where

$$
\begin{aligned}
\left(x_{n 1}(\tau), x_{n 2}(\tau)\right)= & \left(\int_{0}^{1} G_{1}(\tau, \rho) f_{1}\left(\rho, x_{(n-1) 1}(\rho), D_{0^{+}}^{\eta} x_{(n-1) 1}(\rho)\right) d \rho\right. \\
& +\int_{0}^{1} G_{1}(\tau, \rho) g_{1}\left(\rho, y_{(n-1) 1}(\rho)\right) d \rho+\frac{\Gamma(\alpha-\zeta)}{\Gamma(\alpha)} k_{2}\left(x_{(n-1) 1}(1)\right) \tau^{\alpha-1}, \\
& \int_{0}^{1} G_{1}(\tau, \rho) f_{1}\left(\rho, x_{(n-1) 2}(\rho), D_{0^{+}}^{\eta} x_{(n-1) 2}(\rho)\right) d \rho \\
& \left.+\int_{0}^{1} G_{1}(\tau, \rho) g_{1}\left(\rho, y_{(n-1) 2}(\rho)\right) d \rho+\frac{\Gamma(\alpha-\zeta)}{\Gamma(\alpha)} k_{2}\left(x_{(n-1) 2}(1)\right) \tau^{\alpha-1}\right), \\
\left(y_{n 1}(\tau), y_{n 2}(\tau)\right)= & \int_{0}^{1} G_{2}(\tau, \rho) f_{2}\left(\rho, y_{(n-1) 1}(\rho), D_{0^{+}}^{\gamma} y_{(n-1) 1}(\rho)\right) d \rho \\
& +\int_{0}^{1} G_{2}(\tau, \rho) g_{2}\left(\rho, x_{(n-1) 1}(\rho)\right) d \rho+\frac{\Gamma(\beta-\xi)}{\Gamma(\beta)} k_{1}\left(y_{(n-1) 1}(1)\right) \tau^{\beta-1}, \\
& \int_{0}^{1} G_{2}(\tau, \rho) f_{2}\left(\rho, y_{(n-1) 2}(\rho), D_{0^{+}}^{\gamma} y_{(n-1) 2}(\rho)\right) d \rho \\
& \left.+\int_{0}^{1} G_{2}(\tau, \rho) g_{2}\left(\rho, x_{(n-1) 2}(\rho)\right) d \rho+\frac{\Gamma(\beta-\xi)}{\Gamma(\beta)} k_{1}\left(y_{(n-1) 2}(1)\right) \tau^{\beta-1}\right), \\
n & =1,2, \ldots
\end{aligned}
$$

Proof By Lemma 2.6 we have

$$
G_{1}(\tau, \rho), G_{2}(\tau, \rho), D_{0^{+}}^{\eta} G_{1}(\tau, \rho), D_{0^{+}}^{\gamma} G_{1}(\tau, \rho), D_{0^{+}}^{\eta} G_{2}(\tau, \rho), D_{0^{+}}^{\gamma} G_{2}(\tau, \rho) \geq 0
$$

Regarding (16) and $\left(H_{1}\right)$ in (13) we get $A_{1}, A_{2}, B_{1}, B_{2}: P \rightarrow P$ and $C_{1}, C_{2}: P \times P \rightarrow P \times P$.

Obviously $A_{1}, A_{2}$ are increasing and sub-homogeneous, Because $g_{1}, g_{2}$ are increasing and sub-homogeneous. $B_{1}, B_{2}$ are decreasing (due to this fact, $k_{1}$ and $k_{2}$ are decreasing) and satisfy in conditions $B_{i}\left(\lambda^{-1} x\right) \geq \lambda B_{i}(x), i=1,2$, by (15). For any $\left(u_{1}, v_{1}\right),\left(u_{2}, v_{2}\right) \in K$ with $\left(u_{1}, v_{1}\right) \preceq\left(u_{2}, v_{2}\right)$, considering that $f_{1}(\tau, x, y)$ and $f_{2}(\tau, x, y)$ are increasing in $x$ and decreasing in $y$, we have

$$
\begin{array}{llll}
C_{1}\left(v_{1}, u_{1}\right) \leq C_{1}\left(v_{2}, u_{1}\right) & \text { for fixed } u_{1} \quad \text { and } \quad C_{1}\left(v_{1}, u_{1}\right) \geq C_{1}\left(v_{1}, u_{2}\right) & \text { for fixed } v_{1}, \\
C_{2}\left(u_{1}, v_{1}\right) \leq C_{2}\left(u_{2}, v_{1}\right) & \text { for fixed } v_{1} \quad \text { and } \quad C_{2}\left(u_{1}, v_{1}\right) \geq C_{2}\left(u_{1}, v_{2}\right) \text { for fixed } u_{1},
\end{array}
$$

also

$$
C_{1}\left(\tau, \lambda x, \lambda^{-1} y\right) \geq \lambda^{\alpha_{1}} C_{1}(\tau, x, y), \quad C_{2}\left(\tau, \lambda x, \lambda^{-1} y\right) \geq \lambda^{\alpha_{2}} C_{2}(\tau, x, y) .
$$

Set $A=\left(A_{1}, A_{2}\right): K \rightarrow K, B=\left(B_{1}, B_{2}\right): K \rightarrow K, C=\left(C_{1}, C_{2}\right): K \times K \rightarrow K$. Then $A, B, C$ satisfy Eq. (3) of Theorem 2.2, with replacing the cone $K$ for the cone $P$. 
From Lemma 2.7, we get $K_{h}=P_{h_{1}} \times P_{h_{2}}$, where $h(\tau)=\left(h_{1}(\tau), h_{2}(\tau)\right)=\left(\tau^{\alpha-1}, \tau^{\beta-1}\right)$, also by condition $(i)$ of Theorem 2.2, we need prove $A_{1_{h_{1}}}, B_{1_{h_{1}}} \in P_{h_{1}}, A_{2_{h_{2}}}, B_{2_{h_{2}}} \in P_{h_{2}}$ and $C_{1}\left(h_{1}, h_{1}\right) \in P_{h_{1}}, C_{2}\left(h_{2}, h_{2}\right) \in P_{h_{2}}$.

Indeed

$$
\begin{aligned}
A_{1_{h_{1}}}(\tau) & =\int_{0}^{1} G_{1}(\tau, \rho) g_{1}\left(\rho, h_{2}(\rho)\right) d \rho=\int_{0}^{1} G_{1}(\tau, \rho) g_{1}\left(\rho, \rho^{\alpha-1}\right) d \rho \\
& \geq \tau^{\alpha-1} \int_{0}^{1} \frac{(1-\rho)^{\alpha-\zeta-1}\left[1-(1-\rho)^{\zeta}\right]}{\Gamma(\alpha)} g_{1}(\rho, 0) d \rho>0, \\
A_{1_{h_{1}}}(\tau) & =\int_{0}^{1} G_{1}(\tau, \rho) g_{1}\left(\rho, h_{2}(\rho)\right) d \rho=\int_{0}^{1} G_{1}(\tau, \rho) g_{1}\left(\rho, \rho^{\alpha-1}\right) d \rho \\
& \leq \tau^{\alpha-1} \int_{0}^{1} \frac{(1-\rho)^{\alpha-\zeta-1}}{\Gamma(\alpha)} g_{1}(\rho, 1) d \rho .
\end{aligned}
$$

Let

$$
\begin{aligned}
& a_{11}:=\int_{0}^{1} \frac{(1-\rho)^{\alpha-\zeta-1}\left[1-(1-\rho)^{\zeta}\right]}{\Gamma(\alpha)} g_{1}(\rho, 0) d \rho>0 . \\
& a_{12}:=\int_{0}^{1} \frac{(1-\rho)^{\alpha-\zeta-1}}{\Gamma(\alpha)} g_{1}(\rho, 1) d \rho .
\end{aligned}
$$

Then $a_{12} \geq a_{11}>0$ and thus

$$
a_{11} h(\tau) \leq A_{1_{h_{1}}}(\tau) \leq a_{12} h(\tau), \quad 0 \leq \tau \leq 1
$$

Also,

$$
\begin{aligned}
A_{2_{h_{2}}}(\tau) & =\int_{0}^{1} G_{1}(\tau, \rho) g_{2}\left(\rho, h_{1}(\rho)\right) d \rho=\int_{0}^{1} G_{1}(\tau, \rho) g_{2}\left(\rho, \rho^{\beta-1}\right) d \rho \\
& \geq \tau^{\beta-1} \int_{0}^{1} \frac{(1-\rho)^{\beta-\xi-1}\left[1-(1-\rho)^{\xi}\right]}{\Gamma(\beta)} g_{2}(\rho, 0) d \rho>0, \\
A_{2_{h_{2}}}(\tau) & =\int_{0}^{1} G_{1}(\tau, \rho) g_{2}\left(\rho, h_{1}(\rho)\right) d \rho=\int_{0}^{1} G_{1}(\tau, \rho) g_{2}\left(\rho, \rho^{\beta-1}\right) d \rho \\
& \leq \tau^{\beta-1} \int_{0}^{1} \frac{(1-\rho)^{\beta-\xi-1}}{\Gamma(\beta)} g_{2}(\rho, 1) d \rho .
\end{aligned}
$$

Let

$$
\begin{aligned}
& a_{21}:=\int_{0}^{1} \frac{(1-\rho)^{\beta-\xi-1}\left[1-(1-\rho)^{\xi}\right]}{\Gamma(\beta)} g_{1}(\rho, 0) d \rho>0, \\
& a_{22}:=\int_{0}^{1} \frac{(1-\rho)^{\beta-\xi-1}}{\Gamma(\beta)} g_{1}(\rho, 1) d \rho .
\end{aligned}
$$

Then $a_{22} \geq a_{21}>0$ and thus

$$
\begin{aligned}
& a_{21} h(\tau) \leq A_{1_{h_{1}}}(\tau) \leq a_{22} h(\tau), \quad 0 \leq \tau \leq 1, \\
& B_{1}(u)(\tau)=\frac{\Gamma(\alpha-\zeta)}{\Gamma(\alpha)} k_{2}(u(1)) \tau^{\alpha-1}, \quad B_{2}(v)(\tau)=\frac{\Gamma(\beta-\xi)}{\Gamma(\beta)} k_{1}(v(1)) \tau^{\beta-1},
\end{aligned}
$$


therefore

$$
B_{1}\left(h_{1}\right)(\tau)=\frac{\Gamma(\alpha-\zeta)}{\Gamma(\alpha)} k_{2}(1) \tau^{\alpha-1}, \quad B_{2}\left(h_{2}\right)(\tau)=\frac{\Gamma(\beta-\xi)}{\Gamma(\beta)} k_{1}(1) \tau^{\beta-1} .
$$

From $k_{2}(u(1)) \not \equiv 0$ and $k_{1}(v(1)) \not \equiv 0$ we get $B_{1_{h_{1}}} \in P_{h_{1}}, B_{2_{h_{2}}} \in P_{h_{2}}$. We have

$$
\begin{aligned}
C_{1}\left(h_{1}, h_{1}\right)(\tau) & =\int_{0}^{1} G_{1}(\tau, \rho) f_{1}\left(\rho, h(\rho), D_{0^{+}}^{\eta} h(\rho)\right) d \rho \\
& \leq \int_{0}^{1} \frac{\tau^{\alpha-1}(1-\rho)^{\alpha-\zeta-1}}{\Gamma(\alpha)} f_{1}\left(\rho, \rho^{\alpha-1}, \frac{\Gamma(\alpha)}{\Gamma(\alpha-\eta)} \tau^{\alpha-\eta-1}\right) d \rho \\
& \leq \tau^{\alpha-1} \int_{0}^{1} \frac{(1-\rho)^{\alpha-\zeta-1}}{\Gamma(\alpha)} f_{1}(\rho, 1,0) d \rho \\
C_{1}\left(h_{1}, h_{1}\right)(\tau) & =\int_{0}^{1} G_{1}(\tau, \rho) f_{1}\left(\rho, h(\rho), D_{0^{+}}^{\eta} h(\rho)\right) d \rho \\
& \geq \int_{0}^{1} \frac{\tau^{\alpha-1}(1-\rho)^{\alpha-\zeta-1}\left[1-(1-\rho)^{\zeta}\right]}{\Gamma(\alpha)} f_{1}\left(\rho, \rho^{\alpha-1}, \frac{\Gamma(\alpha)}{\Gamma(\alpha-\eta)}(\tau)^{\alpha-\eta-1}\right) d \rho \\
& \geq \tau^{\alpha-1} \int_{0}^{1} \frac{(1-\rho)^{\alpha-\zeta-1}\left[1-(1-\rho)^{\zeta}\right]}{\Gamma(\alpha)} f_{1}\left(\rho, 0, \frac{\Gamma(\alpha)}{\Gamma(\alpha-\eta)}\right) d \rho, \\
C_{2}\left(h_{2}, h_{2}\right)(\tau) & =\int_{0}^{1} G_{2}(\tau, \rho) f_{2}\left(\rho, u(\rho), D_{0^{+}}^{\gamma} \nu(\rho)\right) d \rho \\
& \leq \int_{0}^{1} \frac{\tau^{\beta-1}(1-\rho)^{\beta-\xi-1}}{\Gamma(\beta)} f_{2}\left(\rho, \rho^{\beta-1}, \frac{\Gamma(\beta)}{\Gamma(\beta-\gamma)} \tau^{\beta-\gamma-1}\right) d \rho \\
& \leq \tau^{\beta-1} \int_{0}^{1} \frac{(1-\rho)^{\beta-\xi-1}}{\Gamma(\beta)} f_{2}(\rho, 1,0) d \rho, \\
& \geq \tau^{\beta-1} \int_{0}^{1} \frac{(1-\rho)^{\beta-\xi-1}}{\Gamma(\beta)} f_{2}\left(\rho, 0, \frac{\Gamma(\beta)}{\Gamma(\beta-\gamma)}\right) d \rho . \\
C_{2}\left(h_{2}, h_{2}\right)(\tau) & =\int_{0}^{1} G_{2}(\tau, \rho) f_{2}\left(\rho, u(\rho), D_{0^{+}}^{\gamma} \nu(\rho)\right) d \rho \\
& \geq \int_{0}^{1} \frac{\tau^{\beta-1}(1-\rho)^{\beta-\xi-1}\left[1-(1-\rho)^{\xi}\right]}{\Gamma(\beta)} f_{2}\left(\rho, \rho^{\beta-1}, \frac{\Gamma(\beta)}{\Gamma(\beta-\gamma)}(\tau)^{\beta-\gamma-1}\right) d \rho \\
&
\end{aligned}
$$

We can calculate that

$$
\begin{aligned}
& D_{0^{+}}^{\eta} A_{1}(u)(\tau)=\int_{0}^{1} D_{0^{+}}^{\eta} G_{1}(\tau, \rho) g_{1}(\rho, v(\rho)) d \rho, \\
& D_{0^{+}}^{\gamma} A_{2}(v(\tau))=\int_{0}^{1} D_{0^{+}}^{\gamma} G_{2}(\tau, \rho) g_{2}(\rho, u(\rho)) d \rho, \\
& D_{0^{+}}^{\eta} B_{1}(u)(\tau)=\frac{\Gamma(\alpha-\zeta)}{\Gamma(\alpha-\eta)} k_{2}(u(1)) \tau^{\alpha-\eta-1}, \quad D_{0^{+}}^{\gamma} B_{2}(v)(\tau)=\frac{\Gamma(\beta-\xi)}{\Gamma(\beta-\gamma)} k_{1}(v(1)) \tau^{\beta-\gamma-1}, \\
& D_{0^{+}}^{\eta} C_{1}(v, u)(\tau)=\int_{0}^{1} D_{0^{+}}^{\eta} G_{1}(\tau, \rho) f_{1}\left(\rho, v(\rho), D_{0^{+}}^{\eta} u(\rho)\right) d \rho, \\
& D_{0^{+}}^{\gamma} C_{2}(u, v)(\tau)=\int_{0}^{1} D_{0^{+}}^{\gamma} G_{2}(\tau, \rho) f_{2}\left(\rho, u(\rho), D_{0^{+}}^{\gamma} \nu(\rho)\right) d \rho,
\end{aligned}
$$


also

$$
\begin{aligned}
D_{0^{+}}^{\eta} A_{1}(h)(\tau) & =\int_{0}^{1} D_{0^{+}}^{\eta} G_{1}(\tau, \rho) g_{1}\left(\rho, \rho^{\alpha-1}\right) d \rho \\
& \geq \int_{0}^{1} \frac{\tau^{\alpha-\eta-1}(1-\rho)^{\alpha-\zeta-1}\left[1-(1-\rho)^{\zeta-\eta}\right]}{\Gamma(\alpha-\eta)} g_{1}\left(\rho, \rho^{\alpha-1}\right) d \rho \\
& \geq \tau^{\alpha-\eta-1} \frac{\Gamma(\alpha)}{\Gamma(\alpha-\eta)} \int_{0}^{1} \frac{(1-\rho)^{\alpha-\zeta-1}\left[1-(1-\rho)^{\zeta-\eta}\right]}{\Gamma(\alpha)} g_{1}(\rho, 0) d \rho, \\
D_{0^{+}}^{\eta} A_{1}(h)(\tau) & =\int_{0}^{1} D_{0^{+}}^{\eta} G_{1}(\tau, \rho) g_{1}\left(\rho, \rho^{\alpha-1}\right) d \rho \\
& \leq \int_{0}^{1} \frac{\tau^{\alpha-\eta-1}(1-\rho)^{\alpha-\zeta-1}}{\Gamma(\alpha-\eta)} g_{1}\left(\rho, \rho^{\alpha-1}\right) d \rho \\
& \leq \tau^{\alpha-\eta-1} \frac{\Gamma(\alpha)}{\Gamma(\alpha-\eta)} \int_{0}^{1} \frac{(1-\rho)^{\alpha-\zeta-1}}{\Gamma(\alpha)} g_{1}(\rho, 1) d \rho .
\end{aligned}
$$

Set $a_{11}^{\prime}=\int_{0}^{1} \frac{(1-\rho)^{\alpha-\zeta-1}\left[1-(1-\rho)^{\zeta-\eta}\right]}{\Gamma(\alpha)} g_{1}(\rho, 0) d \rho$ and $a_{12}^{\prime}=\int_{0}^{1} \frac{(1-\rho)^{\alpha-\zeta-1}}{\Gamma(\alpha)} g_{1}(\rho, 1) d \rho$, we have

$$
a_{11}^{\prime} D_{0^{+}}^{\eta} h \leq D_{0^{+}}^{\eta} A_{1}(h) \leq a_{12}^{\prime} D_{0^{+}}^{\eta} h
$$

and by (17) and (18) we have $a_{11}^{\prime} h \leq A_{1}(h) \leq a_{12}^{\prime} h$. So $\min \left\{a_{11}, a_{11}^{\prime}\right\} h \preceq A_{1}(h) \preceq \max \left\{a_{12}\right.$, $\left.a_{12}^{\prime}\right\} h$. Hence $A_{1}(h) \in P_{h}$.

Again we have

$$
\begin{aligned}
D_{0^{+}}^{\gamma} A_{2}(h)(\tau) & =\int_{0}^{1} D_{0^{+}}^{\gamma} G_{1}(\tau, \rho) g_{1}\left(\rho, \rho^{\beta-1}\right) d \rho \\
& \geq \int_{0}^{1} \frac{\tau^{\beta-\gamma-1}(1-\rho)^{\beta-\xi-1}\left[1-(1-\rho)^{\xi-\gamma}\right]}{\Gamma(\beta-\gamma)} g_{1}\left(\rho, \rho^{\beta-1}\right) d \rho \\
& \geq \tau^{\beta-\gamma-1} \frac{\Gamma(\beta)}{\Gamma(\beta-\gamma)} \int_{0}^{1} \frac{(1-\rho)^{\beta-\xi-1}\left[1-(1-\rho)^{\xi-\gamma}\right]}{\Gamma(\beta)} g_{1}(\rho, 0) d \rho, \\
D_{0^{+}}^{\gamma} A_{2}(h)(\tau) & =\int_{0}^{1} D_{0^{+}}^{\gamma} G_{1}(\tau, \rho) g_{1}\left(\rho, \rho^{\beta-1}\right) d \rho \\
& \leq \int_{0}^{1} \frac{\tau^{\beta-\gamma-1}(1-\rho)^{\beta-\xi-1}}{\Gamma(\beta-\gamma)} g_{1}\left(\rho, \rho^{\beta-1}\right) d \rho \\
& \leq \tau^{\beta-\gamma-1} \frac{\Gamma(\beta)}{\Gamma(\beta-\gamma)} \int_{0}^{1} \frac{(1-\rho)^{\beta-\xi-1}}{\Gamma(\beta)} g_{1}(\rho, 1) d \rho .
\end{aligned}
$$

Similarly we set $a_{21}^{\prime}=\int_{0}^{1} \frac{(1-\rho)^{\beta-\xi-1}\left[1-(1-\rho)^{\xi-\eta}\right]}{\Gamma(\beta)} g_{1}(\rho, 0) d \rho$ and $a_{22}^{\prime}=\int_{0}^{1} \frac{(1-\rho)^{\beta-\xi-1}}{\Gamma(\beta)} g_{1}(\rho, 1) d \rho$, we have

$$
a_{21}^{\prime} D_{0^{+}}^{\gamma} h \leq D_{0^{+}}^{\gamma} A_{2}(h) \leq a_{22}^{\prime} D_{0^{+}}^{\gamma} h
$$

and by (17) we have $a_{21}^{\prime} h \leq A_{2}(h) \leq a_{22}^{\prime} h$. So $\min \left\{a_{21}, a_{21}^{\prime}\right\} h \preceq A_{2}(h) \preceq \max \left\{a_{21}, a_{22}^{\prime}\right\} h$, hence $A_{2}(h) \in P_{h}$.

Furthermore,

$$
B_{1}\left(h_{1}\right)=\frac{\Gamma(\alpha-\zeta)}{\Gamma(\alpha)} k_{2}(1) \tau^{\alpha-1}=\frac{\Gamma(\alpha-\zeta)}{\Gamma(\alpha)} k_{2}(1) h_{1}(\tau),
$$




$$
\begin{aligned}
D_{0^{+}}^{\eta} B_{1}\left(h_{1}\right) & =\frac{\Gamma(\alpha-\zeta)}{\Gamma(\alpha-\eta)} k_{2}(1) \tau^{\alpha-\eta-1}=\frac{\Gamma(\alpha)}{\Gamma(\alpha-\eta)} k_{2}(1) \tau^{\alpha-\eta-1} \frac{\Gamma(\alpha-\zeta)}{\Gamma(\alpha)} \\
& =\frac{\Gamma(\alpha)}{\Gamma(\alpha-\eta)} k_{2}(1) D_{0^{+}}^{\eta} h_{1}(\tau),
\end{aligned}
$$

therefore

$$
\begin{aligned}
& B_{2}\left(h_{2}\right)=\frac{\Gamma(\beta-\xi)}{\Gamma(\beta)} k_{1}(1) \tau^{\beta-1}=\frac{\Gamma(\beta-\xi)}{\Gamma(\beta)} k_{1}(1) h_{2}(\tau), \\
& \begin{aligned}
D_{0^{+}}^{\gamma} B_{2}\left(h_{2}\right) & =\frac{\Gamma(\beta-\xi)}{\Gamma(\beta-\gamma)} k_{1}(1) \tau^{\beta-\gamma-1} \frac{\Gamma(\beta)}{\Gamma(\beta-\gamma)} k_{1}(1) \tau^{\beta-\gamma-1} \frac{\Gamma(\beta-\xi)}{\Gamma(\beta)} \\
& =\frac{\Gamma(\beta)}{\Gamma(\beta-\gamma)} k_{1}(1) D_{0^{+}}^{\gamma} h_{2}(\tau),
\end{aligned}
\end{aligned}
$$

from $k_{2}(u(1)) \not \equiv 0$ and $k_{1}(v(1)) \not \equiv 0$ we get $B_{1_{h_{1}}} \in P_{h_{1}}, B_{2_{h_{2}}} \in P_{h_{2}}$.

$$
\begin{aligned}
& D_{0^{+}}^{\eta} C_{1}\left(h_{1}, h_{1}\right)(\tau)=\int_{0}^{1} D_{0^{+}}^{\eta} G_{1}(\tau, \rho) f_{1}\left(\rho, \rho^{\alpha-1}, D_{0^{+}}^{\eta} \rho^{\alpha-1}\right) d \rho \\
& \leq \int_{0}^{1} \frac{\tau^{\alpha-\eta-1}(1-\rho)^{\alpha-\zeta-1}}{\Gamma(\alpha-\eta)} f_{1}\left(\rho, \rho^{\alpha-1}, \frac{\Gamma(\alpha)}{\Gamma(\alpha-\eta)} \tau^{\alpha-\eta-1}\right) d \rho \\
& \leq \frac{\Gamma(\alpha)}{\Gamma(\alpha-\eta)} \tau^{\alpha-\eta-1} \int_{0}^{1} \frac{(1-\rho)^{\alpha-\zeta-1}}{\Gamma(\alpha)} f_{1}(\rho, 1,0) d \rho, \\
& D_{0^{+}}^{\eta} C_{1}\left(h_{1}, h_{1}\right)(\tau)=\int_{0}^{1} D_{0^{+}}^{\eta} G_{1}(\tau, \rho) f_{1}\left(\rho, \rho^{\alpha-1}, D_{0^{+}}^{\eta} \rho^{\alpha-1}\right) d \rho \\
& \geq \int_{0}^{1} \frac{\tau^{\alpha-1}(1-\rho)^{\alpha-\zeta-1}\left[1-(1-\rho)^{\zeta-\eta}\right]}{\Gamma(\alpha)} \\
& \times f_{1}\left(\rho, \rho^{\alpha-1}, \frac{\Gamma(\alpha)}{\Gamma(\alpha-\eta)} \tau^{\alpha-\eta-1}\right) d \rho \\
& \geq \tau^{\alpha-\eta-1} \int_{0}^{1} \frac{(1-\rho)^{\alpha-\zeta-1}\left[1-(1-\rho)^{\zeta-\eta}\right]}{\Gamma(\alpha)} f_{1}\left(\rho, 0, \frac{\Gamma(\alpha)}{\Gamma(\alpha-\eta)}\right) d \rho, \\
& D_{0^{+}}^{\gamma} C_{2}\left(h_{2}, h_{2}\right)(\tau)=\int_{0}^{1} D_{0^{+}}^{\gamma} G_{2}(\tau, \rho) f_{2}\left(\rho, \rho^{\beta-1}, D_{0^{+}}^{\gamma} \rho^{\beta-1}\right) d \rho \\
& \leq \int_{0}^{1} \frac{\tau^{\beta-1}(1-\rho)^{\beta-\xi-1}}{\Gamma(\beta)} f_{2}\left(\rho, \rho^{\beta-1}, \frac{\Gamma(\beta)}{\Gamma(\beta-\gamma)} \tau^{\beta-\gamma-1}\right) d \rho \\
& \leq \tau^{\beta-1} \int_{0}^{1} \frac{(1-\rho)^{\beta-\xi-1}}{\Gamma(\beta)} f_{2}(\rho, 1,0) d \rho, \\
& D_{0^{+}}^{\gamma} C_{2}\left(h_{2}, h_{2}\right)(\tau)=\int_{0}^{1} D_{0^{+}}^{\gamma} G_{2}(\tau, \rho) f_{2}\left(\rho, \rho^{\beta-1}, D_{0^{+}}^{\gamma} \rho^{\beta-1}\right) d \rho \\
& \geq \int_{0}^{1} \frac{\tau^{\beta-1}(1-\rho)^{\beta-\xi-1}\left[1-(1-\rho)^{\xi-\gamma}\right]}{\Gamma(\beta)} \\
& \times f_{2}\left(\rho, \rho^{\beta-1}, \frac{\Gamma(\beta)}{\Gamma(\beta-\gamma)} \tau^{\beta-\gamma-1}\right) d \rho \\
& \geq \tau^{\beta-1} \int_{0}^{1} \frac{(1-\rho)^{\beta-\xi-1}}{\Gamma(\beta)} f_{2}\left(\rho, 0, \frac{\Gamma(\beta)}{\Gamma(\beta-\gamma)}\right) d \rho .
\end{aligned}
$$


Set

$$
\begin{aligned}
& c_{1}=\int_{0}^{1} \frac{(1-\rho)^{\alpha-\zeta-1}\left[1-(1-\rho)^{\alpha-\eta}\right] f_{1}\left(\rho, 0, \frac{\Gamma(\alpha)}{\Gamma(\alpha-\gamma)}\right)}{\Gamma(\alpha)} d \rho, \\
& c_{2}=\int_{0}^{1} \frac{(1-\rho)^{\alpha-\zeta-1}}{\Gamma(\alpha)} f_{1}(\rho, 1,0) d \rho,
\end{aligned}
$$

and

$$
\begin{aligned}
& c_{3}=\int_{0}^{1} \frac{(1-\rho)^{\beta-\xi-1}\left[1-(1-\rho)^{\beta-\gamma}\right] f_{2}\left(\rho, 0, \frac{\Gamma(\beta)}{\Gamma(\beta-\gamma)}\right)}{\Gamma(\beta)} d \rho, \\
& c_{4}=\int_{0}^{1} \frac{(1-\rho)^{\beta-\xi-1}}{\Gamma(\beta)} f_{2}(\rho, 1,0) d \rho .
\end{aligned}
$$

From $\left(H_{2}\right)$ and $\left(H_{4}\right)$, it is clear that

$$
c_{2} \geq c_{1} \geq \delta_{1} a_{11}>0, \quad c_{4} \geq c_{3} \geq \delta_{1} a_{21}>0 .
$$

Consequently,

$$
c_{1} h \preceq C_{1}(h, h) \preceq c_{2} h, \quad c_{3} h \preceq C_{2}(h, h) \preceq c_{4} h .
$$

Next, we show the proof the condition $\left(A_{2}\right)$ of Lemma 2.5. By $\left(H_{4}\right)$,

$$
\begin{aligned}
C_{1}(y, x) & =\int_{0}^{1} G_{1}(\tau, \rho) f_{1}\left(\rho, y(\rho), D_{0^{+}}^{\eta} x(\rho)\right) d \rho \\
& \geq \delta_{11} \int_{0}^{1} G_{1}(\tau, \rho) g_{1}(\rho, y(\rho)) d \rho \\
& =\delta_{11} A_{1}(x) \\
D_{0^{+}}^{\eta} C_{1}(y, x) & =\int_{0}^{1} D_{0^{+}}^{\eta} G_{1}(\tau, \rho) f_{1}\left(\rho, y(\rho), D_{0^{+}}^{\eta} x(\rho)\right) d \rho \\
& \geq \delta_{11} \int_{0}^{1} D_{0^{+}}^{\eta} G_{1}(\tau, \rho) g_{1}(\rho, y(\rho)) d \rho \\
& =\delta_{11} D_{0^{+}}^{\eta} A_{1}(x) .
\end{aligned}
$$

Then $C_{1}(y, x) \succeq \delta_{11} A_{1}(x)$.

$$
\begin{aligned}
C_{2}(x, y) & =\int_{0}^{1} G_{2}(\tau, \rho) f_{2}\left(\rho, x(\rho), D_{0^{+}}^{\gamma} y(\rho)\right) d \rho \\
& \geq \delta_{21} \int_{0}^{1} G_{2}(\tau, \rho) g_{2}(\rho, x(\rho)) d \rho \\
& =\delta_{21} A_{2}(y)
\end{aligned}
$$




$$
\begin{aligned}
D_{0^{+}}^{\gamma} C_{2}(x, y) & =\int_{0}^{1} D_{0^{+}}^{\gamma} G_{2}(\tau, \rho) f_{2}\left(\rho, x(\rho), D_{0^{+}}^{\gamma} y(\rho)\right) d \rho \\
& \geq \delta_{21} \int_{0}^{1} D_{0^{+}}^{\gamma} G_{2}(\tau, \rho) g_{2}(\rho, x(\rho)) d \rho \\
& =\delta_{21} D_{0^{+}}^{\gamma} A_{2}(y) .
\end{aligned}
$$

Then $C_{2}(y, x) \succeq \delta_{21} A_{2}(y)$. From $\left(H_{4}\right)$ and Lemma 2.6, we have

$$
\begin{aligned}
& C_{1}(y, x)= \int_{0}^{1} G_{1}(\tau, \rho) f_{1}\left(\rho, y(\rho), D_{0^{+}}^{\eta} x(\rho)\right) d \rho \\
& \geq \int_{0}^{1} \frac{\tau^{\alpha-1}(1-\rho)^{\alpha-\zeta-1}\left[1-(1-\rho)^{\zeta}\right]}{\Gamma(\alpha)} f_{1}\left(\rho, y(\rho), D_{0^{+}}^{\eta} x(\rho)\right) d \rho \\
& \geq \frac{\tau^{\alpha-1}}{\Gamma(\alpha)}\left(\frac{1}{\alpha-\zeta}-\frac{1}{\alpha}\right) \delta_{12} \\
& \geq \frac{\tau^{\alpha-1}}{\Gamma(\alpha)}\left(\frac{1}{\alpha-\zeta}-\frac{1}{\alpha-\eta}\right) k_{1}(y(1)) \\
&= \frac{1}{\Gamma(\alpha-\zeta)}\left(\frac{1}{\alpha-\zeta}-\frac{1}{\alpha-\eta}\right) B_{2} y, \\
& D_{0^{+}}^{\eta} C_{1}(y, x)=\int_{0}^{1} D_{0^{+}}^{\eta} G(\tau, \rho) f_{1}\left(\rho, y(\rho), D_{0^{+}}^{\eta} x(\rho)\right) d \rho \\
& \quad \geq \frac{\tau^{\alpha-\eta-1}}{\Gamma(\alpha-\eta)} \int_{0}^{1}(1-\rho)^{\alpha-\zeta-1}\left(1-(1-\rho)^{\zeta-\eta}\right) f_{1}\left(\rho, y(\rho), D_{0^{+}}^{\eta} x(\rho)\right) d \rho \\
& \quad \geq \frac{\tau^{\alpha-\eta-1}}{\Gamma(\alpha-\eta)}\left(\frac{1}{\alpha-\zeta}-\frac{1}{\alpha-\eta}\right) k_{1}(y(1)) \\
& \quad=\frac{1}{\Gamma(\alpha-\zeta)}\left(\frac{1}{\alpha-\zeta}-\frac{1}{\alpha-\eta}\right) D_{0^{+}}^{\eta} B_{2} y .
\end{aligned}
$$

That means $C_{1}(x, y) \succeq \frac{1}{\Gamma(\alpha-\zeta)}\left(\frac{1}{\alpha-\zeta}-\frac{1}{\alpha-\eta}\right) B_{2} y$. Let

$$
\delta_{1}=\min \left\{\delta_{12}, \frac{1}{\Gamma(\alpha-\zeta)}\left(\frac{1}{\alpha-\zeta}-\frac{1}{\alpha-\eta}\right)\right\}
$$

then

$$
\begin{aligned}
C_{1}(x, y) & \succeq \delta_{1}\left(A_{1} x+B_{2} y\right), \\
C_{2}(x, y) & =\int_{0}^{1} G_{2}(\tau, \rho) f_{2}\left(\rho, x(\rho), D_{0^{+}}^{\gamma} y(\rho)\right) d \rho \\
& \geq \int_{0}^{1} \frac{\tau^{\beta-1}(1-\rho)^{\beta-\xi-1}\left[1-(1-\rho)^{\xi}\right]}{\Gamma(\beta)} f_{2}\left(\rho, x(\rho), D_{0^{+}}^{\gamma} y(\rho)\right) d \rho \\
& \geq \frac{\tau^{\beta-1}}{\Gamma(\beta)}\left(\frac{1}{\beta-\xi}-\frac{1}{\beta}\right) \delta_{22} \\
& \geq \frac{\tau^{\beta-1}}{\Gamma(\beta)}\left(\frac{1}{\beta-\xi}-\frac{1}{\beta-\gamma}\right) k_{2}(x(1)) \\
& =\frac{1}{\Gamma(\beta-\xi)}\left(\frac{1}{\beta-\xi}-\frac{1}{\beta-\gamma}\right) B_{1} x,
\end{aligned}
$$




$$
\begin{aligned}
D_{0^{+}}^{\gamma} C_{2}(x, y) & =\int_{0}^{1} D_{0^{+}}^{\gamma} G_{2}(\tau, \rho) f_{2}\left(\rho, x(\rho), D_{0^{+}}^{\gamma} y(\rho)\right) d \rho \\
& \geq \frac{\tau^{\beta-\gamma-1}}{\Gamma(\beta-\gamma)} \int_{0}^{1}(1-\rho)^{\beta-\gamma-1}\left(1-(1-\rho)^{\xi-\gamma}\right) f_{2}\left(\rho, x(\rho), D_{0^{+}}^{\gamma} y(\rho)\right) d \rho \\
& \geq \frac{\tau^{\beta-\gamma-1}}{\Gamma(\beta-\gamma)}\left(\frac{1}{\beta-\xi}-\frac{1}{\beta-\gamma}\right) k_{1}(y(1)) \\
& =\frac{1}{\Gamma(\beta-\xi)}\left(\frac{1}{\beta-\xi}-\frac{1}{\beta-\gamma}\right) D_{0^{+}}^{\gamma} B_{1} x .
\end{aligned}
$$

That means $C_{2}(y, x) \succeq \frac{1}{\Gamma(\beta-\xi)}\left(\frac{1}{\beta-\xi}-\frac{1}{\beta-\gamma}\right) B_{1} x$. Let

$$
\delta_{2}=\min \left\{\delta_{22}, \frac{1}{\Gamma(\beta-\xi)}\left(\frac{1}{\beta-\xi}-\frac{1}{\beta-\gamma}\right)\right\} .
$$

Then we have

$$
C_{2}(y, x) \succeq \delta_{2}\left(A_{2} x+B_{1} y\right)
$$

We see that the conclusion (2) in Lemma 2.5 means that there exist $u_{01}, u_{02}, v_{01}, v_{02} \in P_{h}$ and $r \in(0,1)$ such that

(1) $\exists\left(u_{01}, v_{01}\right),\left(u_{02}, v_{02}\right) \in K \subset E \times E$ and $r \in(0,1)$ with

$$
r\left(v_{01}, v_{02}\right) \preceq\left(u_{01}, u_{02}\right) \prec\left(v_{01}, v_{02}\right),
$$

that is,

$$
\begin{aligned}
r\left(v_{01}, v_{02}\right) \leq & \left(u_{01}, u_{02}\right)<\left(v_{01}, v_{02}\right), \\
r\left(D_{0^{+}}^{\gamma} v_{01}, D_{0^{+}}^{\gamma} v_{02}\right) \leq & \left(D_{0^{+}}^{\eta} u_{01}, D_{0^{+}}^{\eta} u_{02}\right)<\left(D_{0^{+}}^{\gamma} v_{01}, D_{0^{+}}^{\gamma} v_{02}\right), \\
\left(u_{01}, u_{02}\right) \leq & \left(\int_{0}^{1} G_{1}(\tau, \rho) f_{1}\left(\rho, u_{01}(\rho), D_{0^{+}}^{\eta} u_{01}(\rho)\right) d \rho\right. \\
& +\int_{0}^{1} G_{1}(\tau, \rho) g_{1}\left(\rho, v_{01}(\rho)\right) d \rho+\frac{\Gamma(\alpha-\zeta)}{\Gamma(\alpha)} k_{2}\left(u_{01}(1)\right) \tau^{\alpha-1}, \\
& \int_{0}^{1} G_{1}(\tau, \rho) f_{1}\left(\rho, u_{02}(\rho), D_{0^{+}}^{\eta} u_{02}(\rho)\right) d \rho \\
& \left.+\int_{0}^{1} G_{1}(\tau, \rho) g_{1}\left(\rho, v_{02}(\rho)\right) d \rho+\frac{\Gamma(\alpha-\zeta)}{\Gamma(\alpha)} k_{2}\left(u_{02}(1)\right) \tau^{\alpha-1}\right), \\
D_{0^{+}}^{\eta}\left(\left(u_{01}, u_{02}\right)\right) \leq & \left(\int_{0}^{1} D_{0^{+}}^{\eta} G_{1}(\tau, \rho) f_{1}\left(\rho, u_{01}(\rho), D_{0^{+}}^{\eta} u_{01}(\rho)\right) d \rho\right. \\
& +\int_{0}^{1} D_{0^{+}}^{\eta} G_{1}(\tau, \rho) g_{1}\left(\rho, v_{01}(\rho)\right) d \rho \\
& +\frac{\Gamma(\alpha-\zeta)}{\Gamma(\alpha)} k_{2}\left(u_{01}(1)\right) \tau^{\alpha-1}, \\
& \int_{0}^{1} D_{0^{+}}^{\eta} G_{1}(\tau, \rho) f_{1}\left(\rho, u_{02}(\rho), D_{0^{+}}^{\eta} u_{02}(\rho)\right) d \rho \\
& \left.+\int_{0}^{1} D_{0^{+}}^{\eta} G_{1}(\tau, \rho) g_{1}\left(\rho, v_{02}(\rho)\right) d \rho+\frac{\Gamma(\alpha-\zeta)}{\Gamma(\alpha)} k_{2}\left(u_{02}(1)\right) \tau^{\alpha-1}\right),
\end{aligned}
$$




$$
\begin{aligned}
\left(v_{01}, v_{02}\right) \geq & \left(\int_{0}^{1} G_{2}(\tau, \rho) f_{2}\left(\rho, v_{01}(\rho), D_{0^{+}}^{\gamma} v_{01}(\rho)\right) d \rho\right. \\
& +\int_{0}^{1} G_{2}(\tau, \rho) g_{2}\left(\rho, u_{01}(\rho)\right) d \rho+\frac{\Gamma(\beta-\xi)}{\Gamma(\beta)} k_{1}\left(v_{01}(1)\right) \tau^{\beta-1}, \\
& \int_{0}^{1} G_{2}(\tau, \rho) f_{2}\left(\rho, v_{02}(\rho), D_{0^{+}}^{\gamma} v_{02}(\rho)\right) d \rho \\
+ & \left.\int_{0}^{1} G_{2}(\tau, \rho) g_{2}\left(\rho, u_{02}(\rho)\right) d \rho+\frac{\Gamma(\beta-\xi)}{\Gamma(\beta)} k_{1}\left(v_{02}(1)\right) \tau^{\beta-1}\right), \\
D_{0^{+}}^{\gamma}\left(v_{01}, v_{02}\right) \geq & \left(\int_{0}^{1} D_{0^{+}}^{\gamma} G_{2}(\tau, \rho) f_{2}\left(\rho, v_{01}(\rho), D_{0^{+}}^{\gamma} v_{01}(\rho)\right) d \rho\right. \\
& +\int_{0}^{1} D_{0^{+}}^{\gamma} G_{2}(\tau, \rho) g_{2}\left(\rho, u_{01}(\rho)\right) d \rho+\frac{\Gamma(\beta-\xi)}{\Gamma(\beta)} k_{1}\left(v_{01}(1)\right) \tau^{\beta-1}, \\
& \quad \int_{0}^{1} D_{0^{+}}^{\gamma} G_{2}(\tau, \rho) f_{2}\left(\rho, v_{02}(\rho), D_{0^{+}}^{\gamma} v_{02}(\rho)\right) d \rho \\
& \left.+\int_{0}^{1} D_{0^{+}}^{\gamma} G_{2}(\tau, \rho) g_{2}\left(\rho, u_{02}(\rho)\right) d \rho+\frac{\Gamma(\beta-\xi)}{\Gamma(\beta)} k_{1}\left(v_{02}(1)\right) \tau^{\beta-1}\right),
\end{aligned}
$$

where $h(\tau)=\left(h_{1}(\tau), h_{2}(\tau)\right)=\left(\tau^{\alpha-1}, \tau^{\beta-1}\right), 0 \leq \tau \leq 1$, and $G_{1}(\tau, \rho), G_{2}(\tau, \rho)$ are defined by (11) and (12), respectively.

(2) The problem (2) has a unique positive solution $\left(u^{*}, v^{*}\right)$ in $K_{h}$;

(3) For $\left(x_{01}, x_{02}\right),\left(y_{01}, y_{02}\right) \in P_{h} \times P_{h}$, there are two iterative sequences $\left\{\left(x_{n 1}, x_{n 2}\right)\right\}$ and $\left\{\left(y_{n 1}, y_{n 2}\right)\right\}$ for approximating $\left(x^{*}, y^{*}\right)$, that is, $\left(x_{n 1}, x_{n 2}\right) \rightarrow\left(x^{*}, y^{*}\right)$ and $\left(y_{n 1}, y_{n 2}\right) \rightarrow\left(x^{*}, y^{*}\right)$, where

$$
\begin{aligned}
\left(x_{n 1}(\tau), x_{n 2}(\tau)\right)= & \left(\int_{0}^{1} G_{1}(\tau, \rho) f_{1}\left(\rho, x_{(n-1) 1}(\rho), D_{0^{+}}^{\eta} x_{(n-1) 1}(\rho)\right) d \rho\right. \\
& +\int_{0}^{1} G_{1}(\tau, \rho) g_{1}\left(\rho, y_{(n-1) 1}(\rho)\right) d \rho+\frac{\Gamma(\alpha-\zeta)}{\Gamma(\alpha)} k_{2}\left(x_{(n-1) 1}(1)\right) \tau^{\alpha-1}, \\
& \int_{0}^{1} G_{1}(\tau, \rho) f_{1}\left(\rho, x_{(n-1) 2}(\rho), D_{0^{+}}^{\eta} x_{(n-1) 2}(\rho)\right) d \rho \\
& \left.+\int_{0}^{1} G_{1}(\tau, \rho) g_{1}\left(\rho, y_{(n-1) 2}(\rho)\right) d \rho+\frac{\Gamma(\alpha-\zeta)}{\Gamma(\alpha)} k_{2}\left(x_{(n-1) 2}(1)\right) \tau^{\alpha-1}\right), \\
\left(y_{n 1}(\tau), y_{n 2}(\tau)\right)= & \int_{0}^{1} G_{2}(\tau, \rho) f_{2}\left(\rho, y_{(n-1) 1}(\rho), D_{0^{+}}^{\gamma} y_{(n-1) 1}(\rho)\right) d \rho, \\
& \int_{0}^{1} G_{2}(\tau, \rho) g_{2}\left(\rho, x_{(n-1) 1}(\rho)\right) d \rho, \frac{\Gamma(\beta-\xi)}{\Gamma(\beta)} k_{1}\left(y_{(n-1) 1}(1)\right) \tau^{\beta-1}, \\
& \int_{0}^{1} G_{2}(\tau, \rho) f_{2}\left(\rho, y_{(n-1) 2}(\rho), D_{0^{+}}^{\gamma} y_{(n-1) 2}(\rho)\right) d \rho, \\
& \left.\int_{0}^{1} G_{2}(\tau, \rho) g_{2}\left(\rho, x_{(n-1) 2}(\rho)\right) d \rho, \frac{\Gamma(\beta-\xi)}{\Gamma(\beta)} k_{1}\left(y_{(n-1) 2}(1)\right) \tau^{\beta-1}\right), \\
& n=1,2, \ldots
\end{aligned}
$$




\subsection{Example}

Let us consider

$$
\left\{\begin{array}{l}
D_{0^{+}}^{\frac{7}{2}} x(\tau)+\tau^{2}+(y(\tau))^{\frac{1}{4}}+(x(\tau))^{\frac{1}{4}}+\left(D_{0^{+}}^{\frac{3}{2}} x(\tau)+1\right)^{-\frac{1}{2}}+1=0, \quad \tau \in(0,1), \\
D_{0^{+}}^{\frac{10}{3}} y(\tau)+\tau+\tau^{3}+\frac{y}{1+y}+\frac{x}{1+x}+\frac{1}{D_{0^{+}}^{\frac{5}{3}}(\tau)+1}=0, \quad \tau \in(0,1), \\
x(0)=x^{\prime}(0)=x^{\prime \prime}(0)=0, \\
y(0)=y^{\prime}(0)=y^{\prime \prime}(0)=0, \\
{\left[D_{0^{+}}^{\frac{8}{5}} x(\tau)\right]_{\tau=1}=(x(1))^{-\frac{1}{3}}+5, \quad\left[D_{0^{+}}^{\frac{11}{6}} y(\tau)\right]_{\tau=1}=\frac{1}{1+y(1)^{\frac{1}{2}}} .}
\end{array}\right.
$$

Let $g_{1}(\tau, y)=(x(\tau))^{\frac{1}{4}}+\tau^{2}, f_{1}(\tau, x, y)=(x(\tau))^{\frac{1}{4}}+(y(\tau)+1)^{-\frac{1}{2}}+1$ and $k_{1}(y)=\frac{1}{1+y^{\frac{1}{2}}}$, also $g_{2}(\tau, x)=\tau^{3}+\frac{x}{1+x}, f_{2}(\tau, x, y)=\tau+\frac{x}{1+x}+\frac{1}{y+1}$ and $k_{2}(x)=x^{-\frac{1}{3}}+5$.

Obviously, $g_{1}, g_{2}:[0,1] \times \mathbb{R}^{+} \rightarrow \mathbb{R}^{+}, f_{1}, f_{2}:[0,1] \times \mathbb{R}^{+} \times \mathbb{R}^{+} \rightarrow \mathbb{R}^{+}$and $k_{1}, k_{2}: \mathbb{R}^{+} \rightarrow \mathbb{R}^{+}$are continuous. It is easy to check that $g_{1}(\tau, y), g_{2}(\tau, x)$ are increasing in $y, x$, respectively, and $k_{1}(y), k_{2}(x)$ are decreasing in $y, x \in \mathbb{R}^{+}$(respectively) and $f_{1}(\tau, x, y), f_{2}(\tau, x, y)$ are increasing in $x$ and decreasing in $y$ for fixed $\tau \in(0,1)$. In addition, for any $\lambda \in(0,1)$ we get

$$
\begin{aligned}
& g_{1}(\tau, \lambda y)=\tau^{2}+(\lambda y(\tau))^{\frac{1}{4}} \geq \lambda^{\frac{1}{4}} 2+\lambda^{\frac{1}{4}}(y(\tau))^{\frac{1}{4}}=\lambda^{\frac{1}{4}} g_{1}(\tau, y), \\
& g_{2}(\tau, \lambda x)=\tau^{3}+\frac{\lambda x}{1+\eta x} \geq \lambda \tau+\lambda \frac{x}{1+x}=\lambda g_{2}(\tau, x), \\
& f_{1}\left(\tau, \lambda x, \lambda^{-1} y\right)=\lambda^{\frac{1}{4}}(x(\tau))^{\frac{1}{4}}+\lambda^{\frac{1}{2}}(y(\tau)+1)^{-\frac{1}{2}}+1 \geq \lambda^{\frac{1}{2}}\left((x(\tau))^{\frac{1}{4}}+\lambda^{\frac{1}{2}}(y(\tau)+1)^{-\frac{1}{2}}+1\right) \\
& =\lambda^{\frac{1}{2}} f(\tau, x, y), \\
& f_{2}\left(\tau, \lambda x, \lambda^{-1} y\right)=\tau+\frac{\lambda x}{1+\lambda x}+\frac{1}{\lambda^{-1} y+1} \geq \tau+\frac{\lambda x}{1+x}+\frac{\lambda}{y+1} \geq \lambda f_{2}(\tau, x, y), \\
& k_{1}\left(\lambda^{-1} y\right)=\frac{1}{1+\left(\lambda^{-1} y\right)^{\frac{1}{2}}} \geq \frac{\lambda^{\frac{1}{2}}}{1+y^{\frac{1}{2}}} \geq \frac{\lambda}{1+y^{\frac{1}{2}}}=\lambda k_{1}(y) . \\
& k_{2}\left(\lambda^{-1} x\right)=\left(\lambda^{-1} x\right)^{-\frac{1}{3}}+5 \geq \lambda^{\frac{1}{3}} k_{2}(x) .
\end{aligned}
$$

Besides, $g_{1}(\tau, 0)=2 \not \equiv 0, g_{2}(\tau, 0)=\tau \not \equiv 0$ Moreover, set $\delta_{1}=\delta_{2}=1$,

$$
\begin{aligned}
& f_{1}(\tau, x, y)=(x(\tau))^{\frac{1}{4}}+(y(\tau)+1)^{-\frac{1}{2}}+1 \geq(x(\tau))^{\frac{1}{4}}+\tau^{2}=\delta_{1} g(\tau, x), \\
& f_{2}(\tau, x, y)=\tau+\frac{x}{1+x}+\frac{1}{y+1} \geq \tau^{3}+\frac{x}{1+x}=\delta_{1} g(\tau, x) .
\end{aligned}
$$

Then by Theorem 3.1 we deduce that $(21)$ has a unique positive solution $\left(x^{*}, y^{*}\right)$ in $\left(P_{h_{1}}, P_{h_{2}}\right)$, where $\left(h_{1}, h_{2}\right)=\left(\tau^{\frac{5}{2}}, \tau^{\frac{7}{3}}\right)$.

\section{Conclusion}

In this manuscript, we extend the existence and uniqueness of positive solutions from a class of fractional differential equations with nonlinear boundary conditions for a new class of coupled system of fractional derivatives. 
Acknowledgements

Not applicable.

Funding

Not applicable

Availability of data and materials

Data sharing not applicable to this article as no datasets were generated or analyzed during the current study.

\section{Competing interests}

The authors declare that they have no competing interests.

\section{Authors' contributions}

All authors read and approved the final manuscript.

\section{Author details}

${ }^{1}$ Department of Mathematics, Faculty of Basic Science, University of Bonab, Bonab, Iran. ${ }^{2}$ Department of Mathematics,

Cankaya University, Ankara, Turkey. ${ }^{3}$ Institute of Space Sciences, Magurele-Bucharest, Romania.

\section{Publisher's Note}

Springer Nature remains neutral with regard to jurisdictional claims in published maps and institutional affiliations.

\section{Received: 13 December 2019 Accepted: 3 March 2020 Published online: 11 March 2020}

\section{References}

1. Afshari, H.: Solution of fractional differential equations in quasi-b-metric and b-metric-like spaces. Adv. Differ. Equ. 2018, 285 (2018)

2. Afshari, H., Kalantari, S., Baleanu, D.: Solution of fractional differential equations via $\alpha-\psi$-Geraghty type mappings. Adv. Differ. Equ. 2018, 347 (2018)

3. Afshari, H., Kheiryan, A.: Tripled fixed point theorems and applications to a fractional differential equation boundary value problem. East Asian Math. J. 10(3), 1750056 (11 pages) (2017). https://doi.org/10.1142/S1793557117500565

4. Afshari, H., Khoshvaghti, L.: The unique solution of some operator equations with an application for fractional differential equations. Bol. Soc. Parana. Mat. (2019, in press). https://doi.org/10.5269/bspm.45296

5. Ahmad, B., Nieto, J.: Existence results for a coupled system of nonlinear fractional differential equations with three-point boundary conditions. Comput. Math. Appl. 58, 1838-1843 (2009)

6. Ahmad, B., Ntouyas, S.K., Alsaedi, A.: On a coupled system of fractional differential equations with coupled nonlocal and integral boundary conditions. Chaos Solitons Fractals 83, 234-241 (2016)

7. Ali, A., Rabiei, F., Shaha, K.: On Ulam's type stability for a class of impulsive fractional differential equations with nonlinear integral boundary conditions. J. Nonlinear Sci. Appl. 10, 4760-4775 (2017). https://doi.org/10.22436/jnsa.010.09.19

8. Ali, A., Shaha, K., Baleanu, D.: Ulam stability results to a class of nonlinear implicit boundary value problems of impulsive fractional differential equations. Adv. Differ. Equ. 2019, 5 (2019). https://doi.org/10.1186/s13662-018-1940-0

9. Aronson, D.G.: A comparison method for stability analysis of nonlinear parabolic problems. SIAM Rev. 20, 245-264 (1978)

10. Arshad, A., Shaha, K., Jarad, F., Gupta, V., Abdeljawad, T.: Existence and stability analysis to a coupled system of implicit type impulsive boundary value problems of fractional-order differential equations. Adv. Differ. Equ. 2019, 101 (2019). https://doi.org/10.1186/s13662-019-2047-y

11. Asma, Arshad, A., Shaha, K., Jarad, F.: Ulam-Hyers stability analysis to a class of nonlinear implicit impulsive fractional differential equations with three point boundary conditions. Adv. Differ. Equ. 2019, 7 (2019). https://doi.org/10.1186/s13662-018-1943-x

12. Bai, C., Fang, J.: The existence of a positive solution for a singular coupled system of nonlinear fractional differential equations. Appl. Math. Comput. 150, 611-621 (2004)

13. Bai, Z., Lü, H.: Positive solutions for boundary value problem of nonlinear fractional differential equation. J. Math. Anal. Appl. 311(2), 495-505 (2005)

14. Baleanu, D., Machado, J.A.T., Luo, A.C.J.: Fractional Dynamics and Control. Springer, Berlin (2012)

15. Cheng, C., Feng, Z., Su, Y.: Positive solutions for boundary value problem of fractional differential equation with derivative terms. Electron. J. Qual. Theory Differ. Equ. 2012, 215, 1-27 (2012)

16. Daftardar-Gejji, V.: Positive solutions of a system of non-autonomous fractional differential equations. J. Math. Anal. Appl. 302, 56-64 (2005)

17. Deng, K.: Global existence and blow-up for a system of heat equations with nonlinear boundary condition. Math. Methods Appl. Sci. 18, 307-315 (1995)

18. Deng, K.: Blow-up rates for parabolic systems. Z. Angew. Math. Phys. 47, 132-143 (1996)

19. Feng, H., Zhai, C.: Existence and uniqueness of positive solutions for a class of fractional differential equation with integral boundary conditions. Nonlinear Anal., Model. Control 22(2), 160-172 (2017)

20. Goodrich, C.S.: Existence of a positive solution to a class of fractional differential equations. Appl. Math. Lett. 23(9), 1050-1055 (2010)

21. Goodrich, C.S.: Existence of a positive solution to systems of differential equations of fractional order. Comput. Math. Appl. 62(3), 1251-1268 (2011)

22. Guo, D.: Fixed points of mixed monotone operators with application. Appl. Anal. 34, 215-224 (1988)

23. Guo, D., Lakskmikantham, V.: Coupled fixed points of nonlinear operators with applications. Nonlinear Anal. 11(5), 623-632 (1987) 
24. Henderson, J., Luca, R.: Positive solutions for a system of fractional differential equations with coupled integral boundary conditions. Appl. Math. Comput. 249, 182-197 (2014)

25. Infante, G.: Nonlocal boundary value problems with two nonlinear boundary conditions. Commun. Appl. Anal. 12, 279-288 (2008)

26. Infante, G., Pietramala, P.: Multiple nonnegative solutions of systems with coupled nonlinear boundary conditions. Math. Methods Appl. Sci. 37, 2080-2090 (2014)

27. Jeli, M., Samet, B.: Existence of positive solutions to an arbitrary order fractional differential equation via a mixed monotone operator method. Nonlinear Anal., Model. Control 20(3), 367-376 (2015)

28. Kilbas, A.A., Srivastava, H.M., Trujillo, J.J.: Theory and Applications of Fractional Differential Equations. North-Holland Mathematics Studies, vol. 204, pp. 7-10 (2006)

29. Liu, L.L., Zhang, X.Q., Liu, L.S., Wu, Y.H.: Iterative positive solutions for singular nonlinear fractional differential equation with integral boundary conditions. Adv. Differ. Equ. 2016, 154 (2016)

30. Pedersen, M., Lin, Z:: Blow-up analysis for a system of heat equations coupled through a nonlinear boundary condition. Appl. Math. Lett. 14, 171-176 (2001)

31. Podlubny, l.: Fractional Differential Equations. Academic Press, New York (1999)

32. Shah, K., Ali, A., Bushnaq, S.: Hyers-Ulam stability analysis to implicit Cauchy problem of fractional differential equations with impulsive conditions. Math. Methods Appl. Sci. 41(17), 8329-8343 (2018). https://doi.org/10.1002/mma.5292

33. Sun, Y., Zhao, M.: Positive solutions for a class of fractional differential equations with integral boundary conditions. Appl. Math. Lett. 34, 17-21 (2014)

34. Wang, H., Zhang, L.: The solution for a class of sum operator equation and its application to fractional differential equation boundary value problems. Bound. Value Probl. 2015, 203 (2015)

35. Xu, X.J., Jiang, D.Q., Yuan, C.J.: Multiple positive solutions to singular positone and semipositone Dirichlet-type boundary value problems of nonlinear fractional differential equations. Nonlinear Anal., Theory Methods Appl. 74(16), 5685-5696 (2011)

36. Yang, C., Zhai, C., Zhang, L.: Local uniqueness of positive solutions for a coupled system of fractional differential equations with integral boundary conditions. Adv. Differ. Equ. 2017, 282 (2017)

37. Yang, W.: Positive solutions for a coupled system of nonlinear fractional differential equations with integral boundary conditions. Comput. Math. Appl. 63(1), 288-297 (2012)

38. Yuan, $C$ : Two positive solutions for $(n-1,1)$-type semigroup integral boundary value problems for coupled systems of nonlinear fractional differential equations. Commun. Nonlinear Sci. Numer. Simul. 18, 858-866 (2013)

39. Yuan, C.J.: Multiple positive solutions for $(n-1,1)$-type semipositone conjugate boundary value problems of nonlinear fractional differential equations. Electron. J. Qual. Theory Differ. Equ. 2010, 36, 1-12 (2010)

40. Zhai, C.B., Yan, W.P., Yang, C.: A sum operator method for the existence and uniqueness of positive solutions to Riemann-Liouville fractional differential equation boundary value problems. Commun. Nonlinear Sci. Numer. Simul. 18, 858-866 (2013)

41. Zhai, C.B., Zhang, L.L.: New fixed point theorems for a mixed monotone operators and local existence-uniqueness of positive solutions for nonlinear boundary value problems. J. Math. Anal. Appl. 382, 594-614 (2011)

42. Zhang, L.L., Tian, H.: Existence and uniqueness of positive solutions for a class of nonlinear fractional differential equations. Adv. Differ. Equ. 2017, 14 (2017)

\section{Submit your manuscript to a SpringerOpen ${ }^{\circ}$ journal and benefit from:}

- Convenient online submission

- Rigorous peer review

- Open access: articles freely available online

- High visibility within the field

- Retaining the copyright to your article

Submit your next manuscript at $\gg$ springeropen.com 